\title{
Visual performance with multifocal soft contact lenses in non-presbyopic myopic eyes during an adaptation period
}

This article was published in the following Dove Press journal:

Clinical Optometry

2I April 2016

Number of times this article has been viewed

\section{Cathleen Fedtke' \\ Klaus Ehrmann ${ }^{1,2}$ \\ Varghese Thomas' \\ Ravi C Bakaraju' ${ }^{1,2}$ \\ 'The Brien Holden Vision Institute, Clinical Trial Research Centre, ${ }^{2} \mathrm{School}$ of Optometry and Vision Science, The University of New South Wales, Sydney, NSW, Australia}

Correspondence: Ravi C Bakaraju The Brien Holden Vision Institute, Clinical Trial Research Centre, Level 4, North Wing, Rupert Myers Building,

Gate I4, Barker Street, UNSW, Sydney,

NSW 2052, Australia

$\mathrm{Tel}+61293857516$

Fax +6I 29385740 I

Email r.bakaraju@brienholdenvision.org
Purpose: Multifocal soft contact lenses (MFCLs) have been proposed and used for controlling the rate of myopia progression; however, little is known on the performance and adaptation with MFCLs in non-presbyopes. This study aims to evaluate the visual performance of four commercially available MFCLs in non-presbyopic myopic eyes during an adaptation period.

Methods: Fifty-two experienced myopic contact lens wearers (67\% female; mean age $21.4 \pm 2.0$ years) were enrolled in this trial and 40 completed the trial. Twenty-six participants (Group 1) wore Lotrafilcon B single vision (SV, control), Omafilcon A MFCL center-distance (D) and center-near $(\mathrm{N})$ and the other 26 participants (Group 2) wore Lotrafilcon B SV, Lotrafilcon B MFCL N, and Balafilcon A MFCL N. Lens order was randomized. Participants wore each allocated lens for a minimum of 8 days over four scheduled visits (dispensing and three follow-up visits) with a 1-week washout period between the lens types. At each visit, high-contrast visual acuity (HCVA) (in logarithm of the minimum angle of resolution [logMAR]) and seven subjective performance variables (via questionnaire) were obtained. Power profiles of each lens type, pupil size, and contact lens centration, with lens placed on the eye, were measured.

Results: The SV control outperformed the MFCLs in all variables $(P<0.05)$. There were no significant differences in HCVA over time, with the exception of monocular HCVA with Omafilcon A MFCL N, which at the end of the adaptation period had significantly $(P<0.05)$ improved by $0.10 \log$ MAR. No differences were found between visits for any subjective variables. Subjectively, Lotrafilcon B MFCL N performed best and was the only lens that did not decenter significantly compared to the SV control. Conversely, Omafilcon A MFCL N was the worst performing and most decentered lens $(P<0.05, \mathrm{y}=-0.39 \mathrm{~mm})$, with the greatest plus area under the power profile.

Conclusion: MFCLs with greatest power variation across the optic zone, a greater plus area under the distance labeled power profile, and/or lenses that were significantly decentered resulted in the lowest subjective ratings. Over time, quality of vision with MFCLs did not change in nonpresbyopic myopic participants, with the exception of Omafilcon A MFCL N, which showed some adaptation effects.

Keywords: multifocal contact lenses, visual performance, contact lens centration, power profiles, non-presbyopes

\section{Introduction}

Myopia, with its increasing prevalence worldwide, ${ }^{1}$ has become a significant global health problem, particularly due to the associated risks of developing glaucoma, ${ }^{2}$ cataract, ${ }^{3}$ and retinal detachment. ${ }^{4}$

Conventionally, myopia is corrected with single vision (SV) spectacle or contact lenses, which provide clear foveal distance vision. Over the last decade, multifocal 
contact lenses (MFCLs) have been proposed and tested as a method of controlling myopia progression.

To date, three hypotheses have been postulated for justifying an intervention with MFCLs. The first approach is based on the associations found between increased accommodative lag and myopia. ${ }^{5-8}$ It was hypothesized that lenses that reduce accommodative lag, that is, on-axis hyperopic defocus, may reduce the rate of myopia progression. It has been shown that lenses incorporated with negative spherical aberration reduce accommodative lag. ${ }^{9}{ }^{10}$ Given that center-near MFCLs (MFCL N) have inherent negative spherical aberration, ${ }^{11}$ it can be argued that they may potentially be producing a therapeutic benefit. The second hypothesis relies on the designs that have shown to reduce the peripheral hyperopic defocus and/or induce peripheral myopic defocus. Such an optical manipulation was proposed to discourage myopia progression. ${ }^{12,13}$ The third hypothesis includes a class of lenses that are designed to impose simultaneous defocus on the visual system, where one zone corrects the refractive error, while the other zone (with relative plus to the baseline prescription power) offers a stop signal (eg, concentric-ring bifocal). ${ }^{14,15}$

All types of MFCLs tested for the reduction of rate of progression reported an efficacy of approximately $35 \%-45 \% .{ }^{14-17}$ On the other hand, the results obtained with bifocal or progressive spectacle lenses based on similar hypothesis remain equivocal. ${ }^{18-20}$ One plausible explanation offered to support results with MFCLs is that perhaps MFCLs deliver a more controlled treatment compared to bifocal or progressive addition spectacle lenses, as the downward ocular deviation with spectacles can significantly reduce the therapeutic effect. ${ }^{21}$

Depending on the optical design, MFCLs can reduce hyperopic defocus, on-axis (eg, MFCL N) or in the periphery of the eye (eg, center-distance MFCL [MFCL D]). Although, the actual theory that underpins the treatment effect still remains questionable, one could infer from the literature that, in general, MFCLs seem to offer a protective effect against myopia progression. One of the main limiting factors for using MFCLs in non-presbyopes is the unwarranted accompaniment of visual compromise. While it is expected from theory that such optics would bring in compromise when compared to SV lenses, there seems to be a prevailing argument that given some time for neural adaptation, the performance is expected to improve. Fernandes et $\mathrm{al}^{22}$ assessed changes in visual performance with MFCL correction in a presbyopic group after 15 days of lens wear and found some adaptation effects. However, to our knowledge no studies have investigated adaptation effects, particularly when MFCLs are prescribed to non-presbyopic individuals.
Further, the majority of the studies investigating therapeutic benefit with MFCLs include custom-designed optics. However, it would be in the practitioner's interest to know the commercially available options at hand, should they be proactive enough to offer MFCLs to their progressing myopic patients. Therefore, we only explored MFCLs available in the market. To date, only MFCL D and concentric-ring bifocal "type" lenses have been explored in the clinical trials that investigated for myopia control. The underlying drive for avoiding MFCL N, albeit its potential to reduce on-axis hyperopic defocus, would have been primarily driven by concern for a presumed greater visual performance degradation than MFCL D and concentric-type MFCLs counterparts. Given that the majority of contact lens manufacturers offer MFCL $\mathrm{N}$ designs, we sought to gauge visual performance of such designs as well.

Although several MFCLs have been tested with respect to their efficacy in slowing myopia progression, ${ }^{14,16,23,24}$ only a few studies (with limited designs) have thoroughly evaluated the visual performance, and none have looked into adaptation with such lenses in myopic non-presbyopic eyes. ${ }^{25-28}$ Lastly, differences in visual performance between MFCLs can be attributed to differences in their power profiles, ${ }^{11,29}$ pupil size, ${ }^{30,31}$ and contact lens centration. . $^{2,32,33}$

Based on this, the objectives of the current study were to assess the visual performance of four commercially available MFCL designs, ie, one MFCL D and three MFCL N, during an adaptation period and to measure and discuss the impact of vision-dependent factors, such as the power profiles, pupil size, and lens centration of the different MFCLs.

\section{Methods \\ Study design and contact lenses}

This was a prospective, participant-masked, multiple-group, cross-over, randomized clinical trial in which participants wore three of five different contact lens types bilaterally. Figure 1 details the outline of the study visits. Participants were randomized into one of two groups. The multiple group design was chosen to avoid discontinuations by participants due to the otherwise extended trial duration. Participants in Group 1 were assigned to wear Lotrafilcon B SV (control), Omafilcon A MFCL D, and Omafilcon A MFCL N, whereas participants in Group 2 were assigned to wear Lotrafilcon B SV (control), Lotrafilcon B MFCL N, and Balafilcon A MFCL N. All MFCLs had high add powers, in order to allow for a direct comparison of visual performance with respect to the lens designs. The Lotrafilcon B SV lens was chosen as a control lens due to its minimal spherical aberration. ${ }^{11}$ The 


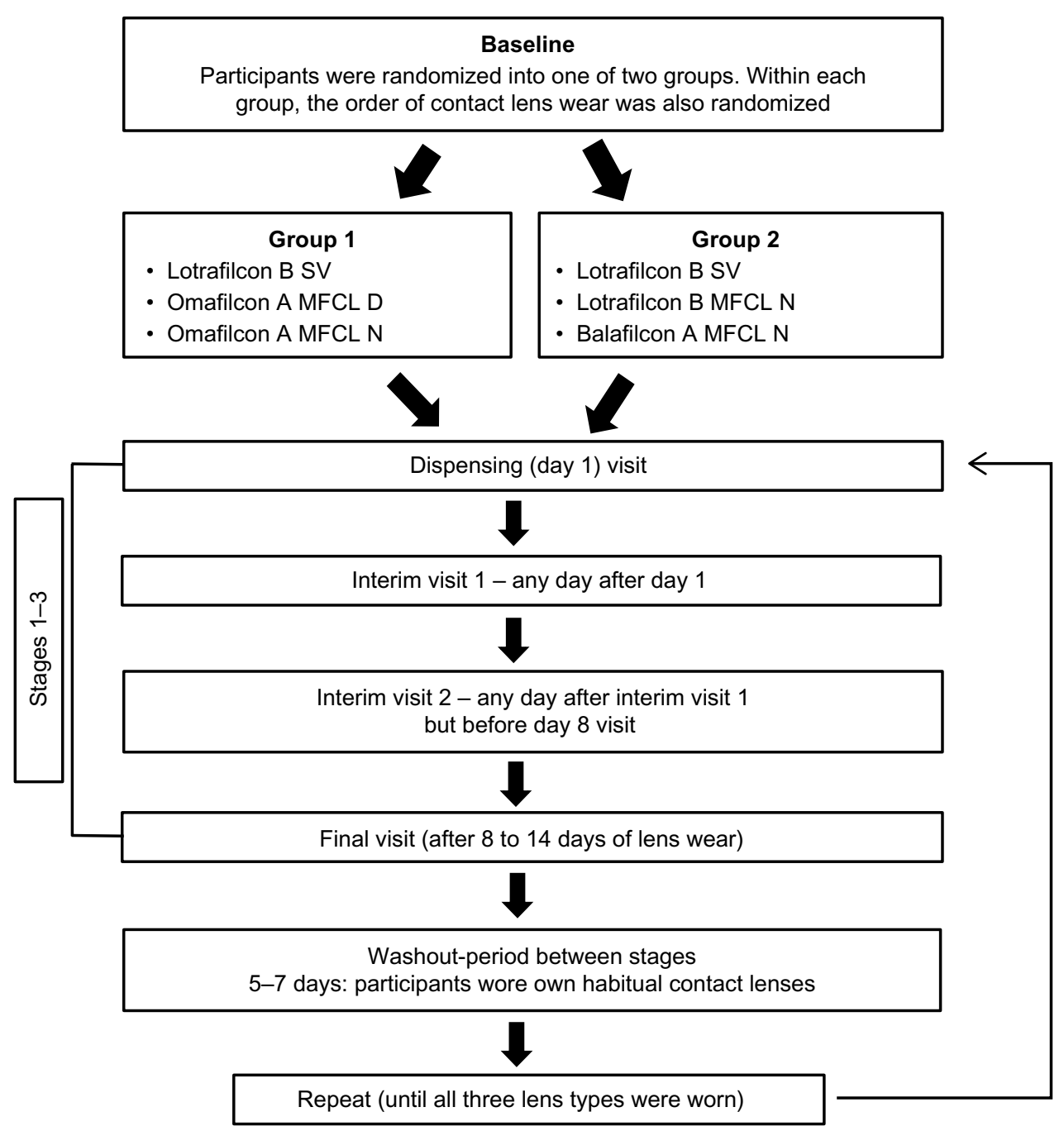

Figure I Outline of study visits.

Abbreviations: SV, single vision; MFCL, multifocal soft contact lens; D, center-distance; N, center-near.

order of lens designs tested in each group was randomized. Table 1 details the lenses used in this trial. AOSept Plus (Alcon, Fort Worth, TX, USA) was used for disinfection and storage of the contact lenses.

The lens power for all lenses was selected based on the spherical equivalent of the distance subjective refraction performed at the baseline visit with the control lens and adjusted for vertex distance. It should be noted that, although using the same distance power for all study lenses permits a direct comparison of visual performance if the lenses were to be fitted as potential myopia control lenses, better vision at distance would be anticipated if an over-refraction was performed, in particular with the MFCL N.

Each participant was asked to wear each of the three lens types on a daily wear schedule for a minimum of 6 hours per day. In total, there were three stages for each participant corresponding to each lens type worn. For each of the three stages, the participant had to attend four visits: a lens dispensing visit, two interim visits, and a final visit. The final visit was performed after 8-14 days of lens wear. The interim visits were performed on any day between day 1 and the final visit of contact lens wear. During all four visits, a series of ocular assessments was performed, which included assessments of monocular and binocular highcontrast visual acuity (HCVA), contact lens fitting, and a questionnaire on vision and comfort with these lenses. To avoid lens bias when answering the questionnaires, the participants were masked to the study contact lenses they wore. The appointments for the two interim visits and the final visit were aimed to be scheduled before $2 \mathrm{pm}$ to avoid taking measurements that might be affected by fatigue of the eyes. At the end of each stage, participants were asked to wear their habitual correction for $5(+2)$ days to allow a washout period before commencing the next stage. 
Table I Description of contact lenses and modality used in both groups

\begin{tabular}{|c|c|c|c|c|c|c|}
\hline Groups & Contact lenses & Design & $\begin{array}{l}\text { Manufacturer, } \\
\text { state, country }\end{array}$ & Material & Diameter (mm) & Base curve $(\mathrm{mm})$ \\
\hline I, 2 & $\begin{array}{l}\text { AIR OPTIX }{ }^{\circledR} \text { AQUA } \\
\text { (control) }\end{array}$ & Single vision & Alcon, TX, USA & Lotrafilcon B & 14.2 & 8.6 \\
\hline \multirow[t]{2}{*}{ I } & $\begin{array}{l}\text { Proclear }^{\circledR} \text { Multifocal } \\
\text { D design }\end{array}$ & $\begin{array}{l}\text { Aspheric center-distance } \\
\text { multifocal }\end{array}$ & $\begin{array}{l}\text { CooperVision, } \\
\text { CA, USA }\end{array}$ & Omafilcon A & 14.4 & 8.7 \\
\hline & $\begin{array}{l}\text { Proclear }^{\circledR} \text { Multifocal } \\
\mathrm{N} \text { design }\end{array}$ & $\begin{array}{l}\text { Aspheric center-near } \\
\text { multifocal }\end{array}$ & $\begin{array}{l}\text { CooperVision, } \\
\text { CA, USA }\end{array}$ & Omafilcon A & 14.4 & 8.7 \\
\hline \multirow[t]{2}{*}{2} & $\begin{array}{l}\text { AIR OPTIX }{ }^{\circledR} \text { AQUA } \\
\text { MULTIFOCAL }\end{array}$ & $\begin{array}{l}\text { Aspheric center-near } \\
\text { multifocal }\end{array}$ & Alcon, TX, USA & Lotrafilcon B & 14.2 & 8.6 \\
\hline & PureVision $^{\circledast}$ Multi-Focal & $\begin{array}{l}\text { Aspheric center-near } \\
\text { multifocal }\end{array}$ & $\begin{array}{l}\text { Bausch and Lomb, } \\
\text { NJ, USA }\end{array}$ & Balafilcon A & 14.0 & 8.6 \\
\hline
\end{tabular}

\section{Participants}

The protocol and informed consent were reviewed and approved by the Bellberry Human Research Ethics Committee, and the research followed the tenets of the Declaration of Helsinki. The study was registered with the Australian and New Zealand Clinical Trial Registry (ACTRN12611001004954) prior to the enrollment of participants. Participants were screened for general trial suitability and written informed consent was obtained prior to any clinical procedures. The inclusion criteria of the study were: myopia between $-1.00 \mathrm{D}$ and $-4.00 \mathrm{D}$, astigmatism no greater than $-1.00 \mathrm{DC}$, and experienced contact lens wearers, aged 18-25 years. Although it would have been preferred to enroll younger participants, ie, an age group in which myopia control therapies are more likely to be tested, previous experience in recruiting young myopes in Sydney proved to be very difficult, and thus the population in this study was limited to young adults. Exclusion criteria included any ocular disease or systemic disease that would contraindicate contact lens wear, or anisometropia above 1.00 D. Fifty-two participants were recruited, of whom 40 participants completed all 12 visits. There were no significant differences $(P>0.05)$ in the number of discontinuations between the groups or lenses. Discontinuations were primarily related to unacceptable vision or discomfort with the study lenses.

\section{Procedures}

Binocular and monocular HCVA (in logarithm of the minimum angle of resolution [logMAR]) was assessed under high illumination (557 \pm 11 lux at participant eye position) using a computer-generated LogMAR vision letter chart (David Thomson Chart 2000; IOO Marketing, London, UK) at a distance of $6 \mathrm{~m} .{ }^{34}$ At each visit, participants rated each lens monocularly for comfort and vision as indicated in Table 2 . The questionnaire used a numeric rating scale, which ranged from 0 to 10 , in steps of 1 unit, where 0 indicates lowest performance and 10 indicates best performance. Pupil sizes were measured at each of the three dispensing visits, under mesopic (80 Lux) illumination condition, using a custombuilt, infrared pupillometer. The power profiles of all study lenses were measured using NIMO TR1504 (Lambda-X, Nivelles, Belgium).

Using a Nikon SLR camera (NIKON FS-3V; Nikon Corporation, Tokyo, Japan) mounted on a slit lamp, photos of the contact lens position post-settling time were taken in straight-ahead gaze at each visit. A custom-written Matlab software was used to determine the amount of $\mathrm{x}$ - and $\mathrm{y}$-contact lens decentration with respect to the pupil center. Positive numbers indicate nasal and superior decentration, while negative numbers indicate temporal and inferior decentration. The exact details of this measurement procedure have been explained previously. ${ }^{35}$

\section{Analysis}

Data were summarized as mean \pm standard deviation for variables measured on an interval scale. Performance variables (HCVA, questionnaire ratings), pupil size, and contact lens centration data were compared between lenses using linear mixed model with subject random intercepts after adjusting for visits. The interaction of lenses with visit was also tested. Post hoc multiple comparisons were adjusted using Bonferroni correction. Analyses were performed separately for the two groups. Level of statistical significance was set at $5 \%$. Analysis was performed using SPSS 21 (IBM Corporation, Armonk, NY, USA).

\section{Results}

A total of 52 participants were enrolled into the study and 40 participants completed each stage of the trial. Thirty-five participants (67\%) were female (female: Group 1: $\mathrm{N}=15$ (58\%), Group 2: $\mathrm{N}=20$ (77\%); $P=0.139)$, the mean age was 
Table 2 Subjective response variables and the corresponding questions asked

\begin{tabular}{ll}
\hline Subjective response variables & Questions \\
\hline Distance vision & "When I look into the distance with my contact lenses, my vision is:" \\
& $\begin{array}{l}\text { Extremely unclear/blurred }=0 \text {, Extremely clear/sharp }=10 \\
\text { "When I look at the TV or computer screen with my contact lenses, my vision is:" } \\
\text { Intermediate vision }\end{array}$ \\
Extremely unclear/blurred $=0$, Extremely clear/sharp $=10$ \\
"When I read books, magazines, and/or newspapers with my contact lenses, my vision is:" \\
Extremely unclear/blurred $=0$, Extremely clear/sharp $=10$
\end{tabular}

21.4 \pm 2.0 years (Group 1: 21.3 \pm 2.0 years, Group 2: 21.5 \pm 2.1 years; $P=0.742$ ), and the mean spherical equivalent obtained via subjective refraction was $-2.82 \pm 0.89 \mathrm{D}$ (Group 1: $-2.92 \pm 0.92 \mathrm{D}$, Group 2: $-2.72 \pm 0.87 \mathrm{D} ; P=0.418)$. There were no significant differences between the groups $(P=0.193)$ or the lens types $(P=0.731)$ with respect to the days the lenses were worn at each of the three follow-up visits. On average, the lenses were worn for $3.8 \pm 2.1$ days at the first interim visit, for $5.3 \pm 2.1$ days at the second interim visit, and for $9.0 \pm 2.4$ days at the final visit.

Table 3 shows the results for binocular and monocular HCVAs as measured with the different lenses of each group at each of the four visits. When compared to all four MFCLs, significantly better results were achieved with the control lens, ie, Lotrafilcon B SV $(P<0.05)$. Omafilcon A MFCL D was the test lens with the overall best HCVA $(P<0.05)$. Group standard deviations were highest for measurements obtained with Omafilcon A MFCL N. Over time, no significant differences $(P>0.05)$ were found between the four visits in both groups for binocular HCVA and in Group 2 for monocular HCVA. There were also no interactions $(P>0.05)$ between lens types and visits. However, there was a significant improvement in monocular HCVA over time with the Omafilcon A MFCL N lens, which was as great as $0.10 \log \mathrm{MAR}$ between the dispensing visit and the final visit.

No statistically significant differences were found for any of the subjective ratings between the visits $(P>0.05)$. Figure 2 shows the mean scores for the ratings obtained on final visit of lens wear in both groups. When compared to control, all MFCLs performed significantly worse in all subjective ratings. Among all the MFCLs, the Lotrafilcon B MFCL N lens achieved the overall highest ratings and Omafilcon A MFCL N was the overall worst performing lens. Based on paired differences to the control lens, Lotrafilcon B MFCL $\mathrm{N}$ lens was rated significantly higher than Omafilcon A MFCL $N$ for distance, intermediate and near $(P<0.05)$.

The mesopic pupil sizes with MFCLs were not significantly different from those obtained with the SV lens $(P>0.05)$. This observation was noticed in both the groups. In Group 1, patients wearing Lotrafilcon B SV, Omafilcon A MFCL D, and Omafilcon A MFCL $N$ had average pupil sizes of $4.7 \pm 1.1$,

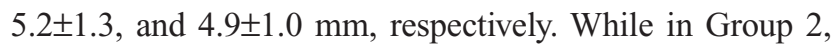
patients wearing Lotrafilcon B SV, Lotrafilcon B MFCL N, and Balafilcon A MFCL $N$ were found to have average pupil sizes of $4.4 \pm 1.1,4.5 \pm 0.9$, and $4.6 \pm 0.8 \mathrm{~mm}$, respectively.

Figure 3 shows the average contact lens centration data for the different lens types. While there were no significant differences in Group 1 with respect to $\mathrm{x}$-centration of the lens, in Group 2, the Balafilcon A MFCL N lens was slightly but significantly decentered toward the temporal side $(\mathrm{x}=-0.05 \mathrm{~mm}$, $P<0.05)$ when compared to the control (x=0.01 mm). With the exception of the Lotrafilcon B MFCL N lens $(P>0.05)$, all other test lenses were significantly inferiorly decentered $(P<0.05)$, when compared to the control. On average, the greatest $y$-decentration of $-0.39 \mathrm{~mm}$ was observed for the Omafilcon A MFCL $\mathrm{N}$ lens. Linear mixed model showed no significant differences $(P>0.05)$ between visits in terms of contact lens position, and there were also no interactions between lens types and visits. All subjective ratings showed a small but significant positive correlation $(r>0.10, P<0.05)$ with y-decentration, indicating a decrease in visual performance as $y$-decentration became more negative.

The power profiles of the different study lenses are shown in Figure 4, indicating the greatest power variations across the optic zone for the Omafilcon A MFCLs. Table 4 lists 
Table 3 Binocular and monocular high-contrast visual acuity (logMAR) as measured with the different lenses at all visits

\begin{tabular}{|c|c|c|c|c|c|c|c|c|c|c|c|c|c|}
\hline \multirow[t]{2}{*}{ Variables } & \multirow[t]{2}{*}{ Group } & \multirow[t]{2}{*}{ Lens } & \multicolumn{2}{|c|}{$\begin{array}{l}\text { Dispensing } \\
\text { visit }\end{array}$} & \multicolumn{2}{|c|}{$\begin{array}{l}\text { Interim } \\
\text { visit I }\end{array}$} & \multicolumn{2}{|c|}{$\begin{array}{l}\text { Interim } \\
\text { visit } 2\end{array}$} & \multicolumn{2}{|l|}{$\begin{array}{l}\text { Final } \\
\text { visit }\end{array}$} & \multicolumn{3}{|c|}{ Linear mixed model } \\
\hline & & & Mean & SD & Mean & SD & Mean & SD & Mean & SD & Lens & Visit & Interaction \\
\hline \multirow{12}{*}{$\begin{array}{l}\text { Binocular } \\
\text { HCVA }\end{array}$} & Group I & Lotrafilcon & -0.13 & 0.06 & -0.14 & 0.04 & -0.13 & 0.06 & -0.13 & 0.06 & $<0.00 I^{*}$ & 0.445 & 0.106 \\
\hline & & $B-S V$ & & & & & & & & & & & \\
\hline & & Omafilcon & -0.11 & 0.06 & -0.12 & 0.06 & -0.10 & 0.06 & -0.10 & 0.06 & & & \\
\hline & & $A-M F C L D$ & & & & & & & & & & & \\
\hline & & Omafilcon & 0.04 & 0.12 & 0.01 & 0.12 & -0.01 & 0.11 & -0.03 & 0.11 & & & \\
\hline & & $A-M F C L N$ & & & & & & & & & & & \\
\hline & Group 2 & Lotrafilcon & -0.13 & 0.07 & -0.13 & 0.06 & -0.13 & 0.05 & -0.14 & 0.05 & $<0.00 I^{*}$ & 0.274 & 0.728 \\
\hline & & $B-S V$ & & & & & & & & & & & \\
\hline & & Lotrafilcon & -0.07 & 0.07 & -0.05 & 0.06 & -0.06 & 0.05 & -0.06 & 0.06 & & & \\
\hline & & $B-M F C L N$ & & & & & & & & & & & \\
\hline & & Balafilcon & -0.03 & 0.08 & 0.00 & 0.08 & -0.02 & 0.08 & -0.01 & 0.08 & & & \\
\hline & & $A-M F C L N$ & & & & & & & & & & & \\
\hline \multirow{12}{*}{$\begin{array}{l}\text { Monocular } \\
\text { HCVA }\end{array}$} & Group I & Lotrafilcon & -0.07 & 0.06 & -0.07 & 0.07 & -0.06 & 0.07 & -0.08 & 0.06 & $<0.00 I^{*}$ & $0.004^{*}$ & $0.007^{*}$ \\
\hline & & $B-S V$ & & & & & & & & & & & \\
\hline & & Omafilcon & -0.02 & 0.13 & -0.02 & 0.09 & -0.03 & 0.09 & -0.03 & 0.09 & & & \\
\hline & & $A-M F C L D$ & & & & & & & & & & & \\
\hline & & Omafilcon & 0.13 & 0.15 & 0.06 & 0.11 & 0.05 & 0.12 & 0.03 & 0.10 & & & \\
\hline & & $A-M F C L N$ & & & & & & & & & & & \\
\hline & Group 2 & Lotrafilcon & -0.08 & 0.08 & -0.09 & 0.07 & -0.06 & 0.08 & -0.07 & 0.07 & $<0.00 I^{*}$ & 0.126 & 0.087 \\
\hline & & $B-S V$ & & & & & & & & & & & \\
\hline & & Lotrafilcon & 0.00 & 0.08 & 0.04 & 0.09 & 0.01 & 0.07 & 0.02 & 0.07 & & & \\
\hline & & $B-M F C L N$ & & & & & & & & & & & \\
\hline & & Balafilcon & 0.04 & 0.08 & 0.06 & 0.09 & 0.05 & 0.07 & 0.06 & 0.08 & & & \\
\hline & & $A-M F C L N$ & & & & & & & & & & & \\
\hline
\end{tabular}

Note: *Statistically significant at an alpha level of 0.05. A linear mixed model was used.

Abbreviations: HCVA, high-contrast visual acuity; SD, standard deviation; SV, single vision; MFCL, multifocal soft contact lens; D, center-distance; N, center-near; logMAR, logarithm of the minimum angle of resolution.

the fourth-order polynomials that were fitted to the power profiles and the calculated corresponding areas under the distance labeled power profiles between the half chords of 0.00 and $3.00 \mathrm{~mm}$. Although the greatest plus area under the distance labeled power profile was found with the Omafilcon A MFCL N lens, the area of the Lotrafilcon B MFCL N lens comprised the least plus power of all MFCLs.

\section{Discussion}

The current study showed that for the HCVAs and subjective visual performance variables, the SV control lens was superior when compared to the four MFCLs (one MFCL D and three MFCL N designs). Similarly, previous studies in non-presbyope ${ }^{25-27}$ also reported some reduction in vision with MFCLs but the results differed between MFCL D and MFCL N designs. Specifically, when testing a concentric bifocal lens with a central distance zone (Acuvue Bifocal), the study by Shah and Gunde ${ }^{27}$ found a significant reduction in low-contrast VA, but not in HCVA when compared to an SV lens. Likewise, Kollbaum et al ${ }^{26}$ reported good HCVA but reduced visual quality ratings with the two test lenses, as both featured a central distance zone. Conversely, with MFCL N designs, Montés-Micó et a ${ }^{25}$ found a significant reduction in high-contrast VA when compared to an SV lens. In agreement with previous studies, a better HCVA was also achieved with the MFCL D lens (Omafilcon A MFCL D) used in the current study when compared to the three MFCL N lenses. The findings on good HCVA with MFCL D lenses can primarily be explained by the fact that it is easier to resolve fine details at distance with a central distance zone lens than when using the peripheral distance zone of a center-near lens. Nevertheless, the overall best performing lens with respect to the subjective response ratings (distance, intermediate, and near) was a center-near lens, ie, the Lotrafilcon B MFCL N lens. This suggests that good distance visual acuity alone does not necessarily imply good overall visual performance in non-presbyopes.

The differences in overall visual performance between the lenses can mainly be attributed to the power profile differences between the lenses and the magnitude of the plus area under the distance labeled power profiles. While the power profiles of the Omafilcon A MFCL D lenses show 

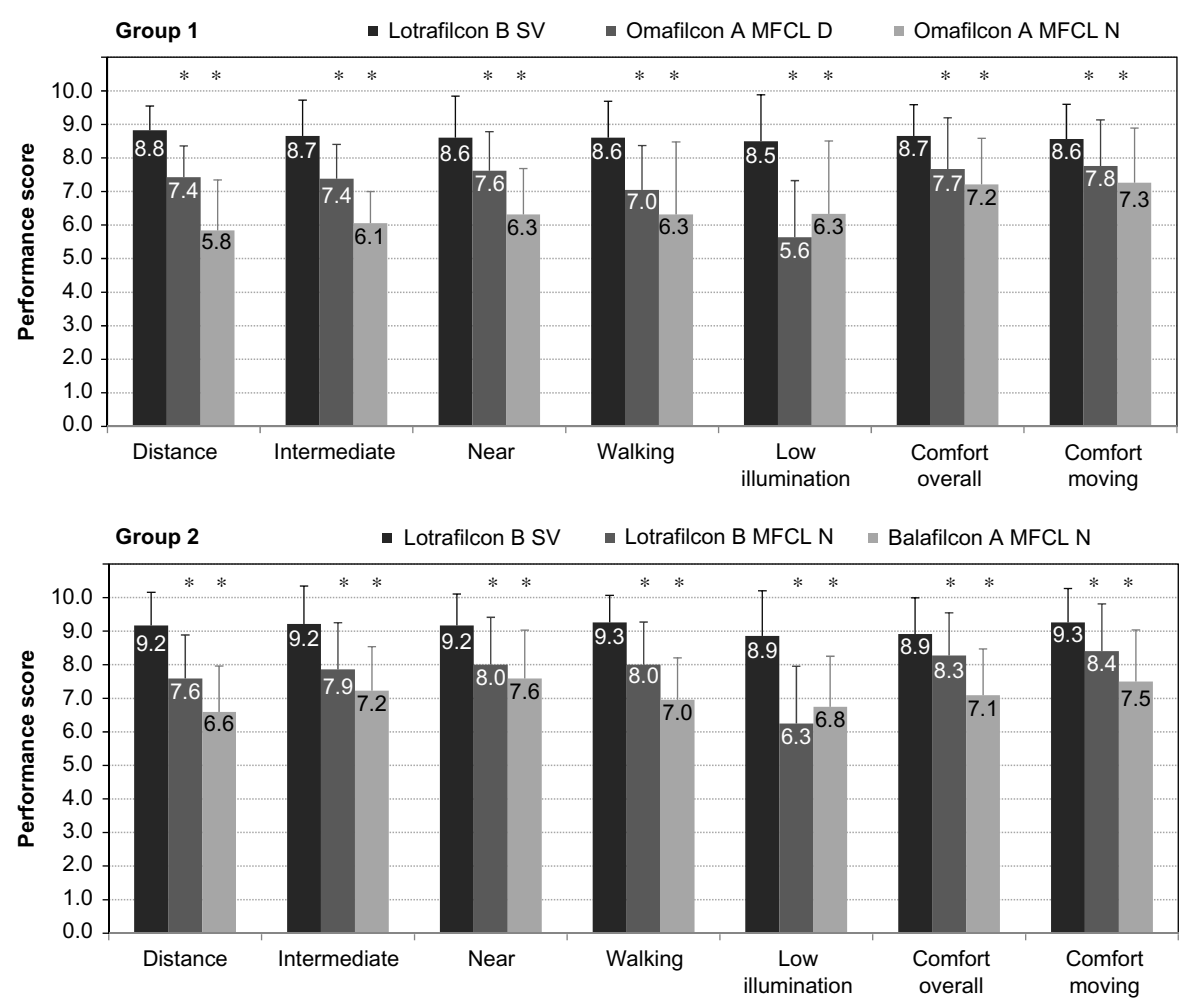

Figure 2 Mean scores for the subjective responses obtained on the final visit of lens wear.

Notes: A linear mixed model was used. Error bars indicate standard deviations of the group; *indicate significant differences $(P<0.05)$ when compared to the control lens (Lotrafilcon B SV), subjective response scores were measured according to the following references; with distance, intermediate, near and low illumination vision and vision while walking scored according to the following; extremely unclear/blurred $=0$, extremely clear/sharp $=10$, whereas overall comfort and comfort moving were measured according to the following references; very uncomfortable $=0$, very comfortable $=10$.

Abbreviations: SV, single vision; MFCL, multifocal soft contact lens; D, center-distance; N, center-near.

a distinct jump in the power profile, a more gradual and smooth power transition across the optical zone occurs with the Lotrafilcon B MFCL N and the Balafilcon A MFCL N lenses. ${ }^{11}$ As noted previously, ${ }^{28}$ the greater the refractive power variations across the optical zone, the greater the magnitudes of higher-order aberrations and, thus, the impact of ghosting. The level of ghosting not only depends on the lens design (ie, power profiles), but also on the near add power, pupil size, and contact lens centration. ${ }^{28}$ The latter factor is supported by the findings of the current study, as Lotrafilcon B MFCL N subjectively performed the best and was the only lens not significantly decentered when compared to the control. On the contrary, Omafilcon A MFCL N was the worst performing and most inferiorly decentered lens.
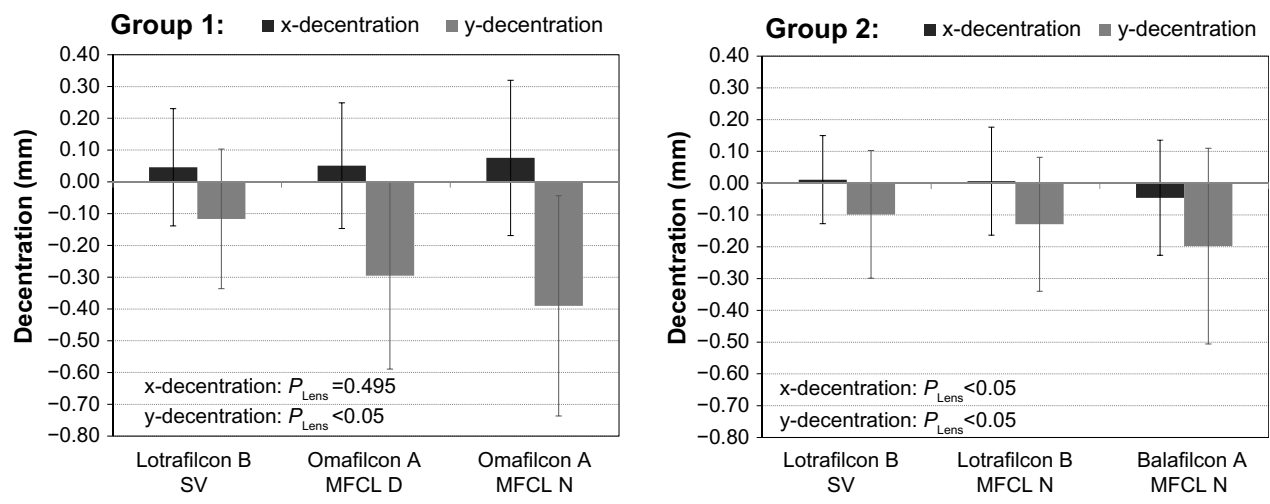

Figure 3 Contact lens centration data (average of four visits).

Notes: A Linear mixed model was used, results are statistically significant at an alpha level of 0.05 .

Abbreviations: SV, single vision; MFCL, multifocal soft contact lens; D, center-distance; N, center-near. 


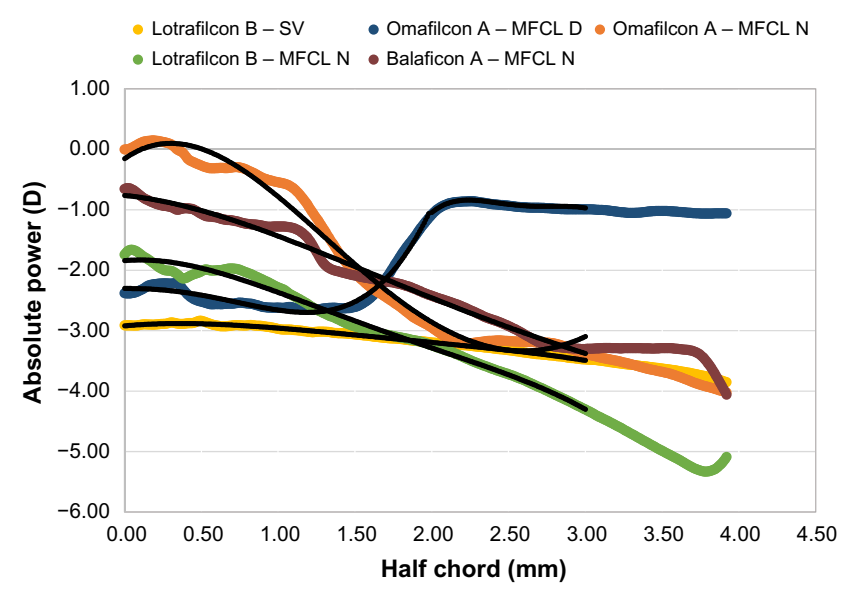

Figure 4 NIMO power profile measurements for all study lenses (distance power label $-3.00 \mathrm{D}$, high add).

Notes: The power profiles of all study lenses were measured using NIMO TRI504 (Lambda-X, Nivelles, Belgium).

Abbreviations: SV, single vision; MFCLD, multifocal soft contact lens centerdistance; MFCLN, multifocal soft contact lens center-near.

Significant inferior contact lens decentration with Omafilcon A MFCLs has been reported previously ${ }^{28,35}$ and may be due to a general looser fit with those lenses. It has been shown that a significant decentration can lead to an increase in odd higher-order aberrations, ${ }^{28}$ which could have adversely affected the visual performance. Overall, the results of the current study suggest that MFCLs with the greatest refractive power variations across the optical zone, and/or being significantly decentered, resulted in the lowest overall visual performance in non-presbyopic myopic eyes. These findings should be kept in mind when practitioners attempt to fit nonpresbyopes with MFCLs.

Finally, the current study is the first study to concomitantly report assessment of visual performance changes with MFCLs over time in myopic non-presbyopes. Unlike the study with presbyopes by Papas et al, ${ }^{36}$ which reported a significant reduction in subjective vision responses over 4 days of MFCL wear, no significant changes in the subjective performance variables were found over a minimum of
8 days of lens wear with the multifocal lenses used in the current study. However, a clinically and statistically significant improvement in monocular distance HCVA was achieved with the Omafilcon A MFCL N lens, which is in agreement with the study by Fernandes et $\mathrm{al},{ }^{22}$ who assessed the same lens design in presbyopes over 15 days of lens wear. It is possible that the size of the plus area under the distance labeled power profile required more time from the participant to adapt to this lens type.

The exact duration needed for a complete neuroadaptation to MFCLs has been debated for decades. The opinions vary from a few days to several weeks; $;^{22,36,37}$ however, recent data on presbyopic individuals suggest that 3-4 days of MFCL wear should be sufficient. ${ }^{36,37}$ However, all the studies investigating adaptation with MFCLs were done on presbyopes. Our current findings from a non-presbyopic sample suggest that the adaptation, if any, would occur in a couple of days. It is worthwhile to note that despite the fact that some adaptation effect was found for monocular HCVA with the Omafilcon MFCL N lens, there was no change in any of the subjective performance variables with this or any other test lens. This constant subjective performance suggests that over time improvement in HCVA perhaps did not impact the participants' daily visual tasks. Based on this observation, and as reported previously, ${ }^{26,28,36}$ subjective measures appear to be more realistic indicators for the assessment of visual performance with MFCLs, when compared to acuity-based measures.

\section{Conclusion}

MFCLs with greatest power variation across the optic zone, a greater plus area under the distance labeled power profile, and/or lenses that were significantly decentered resulted in the lowest subjective ratings. Over time, quality of vision with MFCLs did not change in non-presbyopic myopic participants, with the exception of Omafilcon A MFCL N, which showed some adaptation effects.

Table 4 Fourth-order polynomial fits for the different study lenses and the calculated area under the distance corrected power profiles between the half chords of 0.00 and $3.00 \mathrm{~mm}$

\begin{tabular}{llll}
\hline Lens name & Fourth-order fit & $\boldsymbol{R}^{2}$ & Area \\
\hline Lotrafilcon B SV & $Y_{0-3 \mathrm{~mm}}=-0.0203 x^{4}+0.1448 x^{3}-0.3919 x^{2}+0.2300 x-2.9176$ & 0.9968 & -0.2993 \\
Lotrafilcon B MFCL N & $y_{0-3 \mathrm{~mm}}=-0.0751 x^{4}+0.5003 x^{3}-1.1718 x^{2}+0.2206 x-1.8386$ & 0.9886 & 0.4119 \\
Omafilcon A MFCL D & $Y_{0-2 \mathrm{~mm}}=0.3398 x^{4}-0.3188 x^{3}-0.3920 x^{2}+0.0136 x-2.2997$ & 0.9659 & 3.3716 \\
& $Y_{2-3 \mathrm{~mm}}=-3.4633 x^{4}+36.15 x^{3}-140.78 x^{2}+242.24 x-156.19$ & 0.9829 & 3.7765 \\
Omafilcon A MFCL N & $y_{0-3 \mathrm{~mm}}=-0.0976 x^{4}+1.1326 x^{3}-3.4366 x^{2}+1.7708 x-0.1515$ & 0.9863 & 3.0868 \\
Balafilcon A MFCL N & $y_{0-3 \mathrm{~mm}}=-0.0158 x^{4}+0.1697 x^{3}-0.5720 x^{2}-0.2560 x-0.7606$ & 0.9880 &
\end{tabular}

Abbreviations: SV, single vision; MFCL, multifocal soft contact lens; $\mathrm{D}$, center-distance; $\mathrm{N}$, center-near. 


\section{Acknowledgment}

This work was carried out at the clinics and laboratories of the Brien Holden Vision Institute, Sydney, Australia. The research was fully funded by the Brien Holden Vision Institute. The authors would like to acknowledge the clinical team (Ms Robertson, Ms Crompton, Ms Chung, and Mr Ozkan) and the database management team (Dr Naduvilath and Ms Laarakkers) for their invaluable support to run the study at the Clinical Trial Research Centre, Brien Holden Vision Institute, Sydney, Australia. Authors would like to extend their thanks to Dr Flanagan for reviewing the manuscript and to Dr Kim for measuring the power profiles of the study lenses.

\section{Disclosure}

The Brien Holden Vision Institute receives royalties from the sale of Lotrafilcon B MFCL N. The authors report no other conflicts of interest in this work.

\section{References}

1. Bar Dayan Y, Levin A, Morad Y, et al; The changing prevalence of myopia in young adults: a 13-year series of population-based prevalence surveys. Invest Ophthalmol Vis Sci. 2005;46:2760-2765.

2. Perera SA, Wong TY, Tay WT, Foster PJ, Saw SM, Aung T. Refractive error, axial dimensions, and primary open-angle glaucoma: the Singapore Malay Eye Study. Arch Ophthalmol. 2010;128:900-905.

3. Wong TY, Klein BE, Klein R, Tomany SC, Lee KE. Refractive errors and incident cataracts: the Beaver Dam Eye Study. Invest Ophthalmol Vis Sci. 2001;42:1449-1454.

4. Baba T, Ohno-Matsui K, Futagami S, et al; Prevalence and characteristics of foveal retinal detachment without macular hole in high myopia. Am J Ophthalmol. 2003;135:338-342.

5. Gwiazda JE, Hyman L, Norton TT, et al; Accommodation and related risk factors associated with myopia progression and their interaction with treatment in COMET children. Invest Ophthalmol Vis Sci. 2004;45:2143-2151.

6. Gwiazda J, Thorn F, Bauer J, Held R. Myopic children show insufficient accommodative response to blur. Invest Ophthalmol Vis Sci. 1993;34:690-694.

7. Mutti DO, Mitchell GL, Hayes JR, et al; Accommodative lag before and after the onset of myopia. Invest Ophthalmol Vis Sci. 2006;47:837-846.

8. Abbott ML, Schmid KL, Strang NC. Differences in the accommodation stimulus response curves of adult myopes and emmetropes. Ophthalmic Physiol Opt. 1998;18:13-20.

9. Gambra E, Sawides L, Dorronsoro C, Marcos S. Accommodative lag and fluctuations when optical aberrations are manipulated. $J$ Vis. 2009;9:4.

10. Theagarayan B, Radhakrishnan H, Allen PM, Calver RI, Rae SM, O'Leary DJ. The effect of altering spherical aberration on the static accommodative response. Ophthalmic Physiol Opt. 2009;29: 65-71.

11. Wagner S, Conrad F, Bakaraju RC, Fedtke C, Ehrmann K, Holden B. Power profiles of single vision and multifocal soft contact lenses. Cont Lens Anterior Eye. 2014;38:2-14.

12. Smith EL, 3rd, Kee CS, Ramamirtham R, Qiao-Grider Y, Hung LF. Peripheral vision can influence eye growth and refractive development in infant monkeys. Invest Ophthalmol Vis Sci. 2005;46: 3965-3972.
13. Mutti DO, Hayes JR, Mitchell GL, et al; Refractive error, axial length, and relative peripheral refractive error before and after the onset of myopia. Invest Ophthalmol Vis Sci. 2007;48:2510-2519.

14. Anstice NS, Phillips JR. Effect of dual-focus soft contact lens wear on axial myopia progression in children. Ophthalmology. 2011;118:1152-1161.

15. Lam CS, Tang WC, Tse DY, Tang YY, To CH. Defocus Incorporated Soft Contact (DISC) lens slows myopia progression in Hong Kong Chinese schoolchildren: a 2-year randomised clinical trial. British Journal of Ophthalmology. 2014;98:40-45.

16. Sankaridurg P, Holden B, Smith E 3rd, et al; Decrease in rate of myopia progression with a contact lens designed to reduce relative peripheral hyperopia: one-year results. Invest Ophthalmol Vis Sci. 2011;52:9362-9367.

17. Walline JJ, Greiner KL, McVey ME, Jones-Jordan LA. Multifocal contact lens myopia control. Optom Vis Sci. 2013;90:1207-1214.

18. Edwards MH, Li RW, Lam CS, Lew JK, Yu BS. The Hong Kong progressive lens myopia control study: study design and main findings. Invest Ophthalmol Vis Sci. 2002;43:2852-2858.

19. Gwiazda J, Hyman L, Hussein M, et al; A randomized clinical trial of progressive addition lenses versus single vision lenses on the progression of myopia in children. Invest Ophthalmol Vis Sci. 2003;44:1492-1500.

20. Leung JT, Brown B. Progression of myopia in Hong Kong Chinese schoolchildren is slowed by wearing progressive lenses. Optom Vis Sci. 1999;76:346-354.

21. Hasebe S, Nakatsuka C, Hamasaki I, Ohtsuki H. Downward deviation of progressive addition lenses in a myopia control trial. Ophthalmic Physiol Opt. 2005;25:310-314.

22. Fernandes PR, Neves HI, Lopes-Ferreira DP, Jorge JM, GonzalezMeijome JM. Adaptation to multifocal and monovision contact lens correction. Optom Vis Sci. 2013;90:228-235.

23. Aller TA, Wildsoet C. Bifocal soft contact lenses as a possible myopia control treatment: a case report involving identical twins. Clin Exp Optom. 2008;91:394-399.

24. Walline JJ, McVey L. Myopia control with a soft bifocal contact lens. Optom Vis Sci. 2011;88:395-403.

25. Montés-Micó R, Madrid-Costa D, Radhakrishnan H, Charman WN, Ferrer-Blasco T. Accommodative functions with multifocal contact lenses: a pilot study. Optom Vis Sci. 2011;88:998-1004.

26. Kollbaum PS, Jansen ME, Tan J, Meyer DM, Rickert ME. Vision performance with a contact lens designed to slow myopia progression. Optom Vis Sci. 2013;90:205-214.

27. Shah AS, Gundel R. Low-contrast visual acuity measurements in single-vision and bifocal soft lens wearers. Int Cont Lens Clin. 2000;27:119-123.

28. Fedtke C, Bakaraju RC, Ehrmann K, Chung J, Thomas V, Holden B. Visual performance of single vision and multifocal contact lenses in nonpresbyopic myopic eyes. Cont Lens Anterior Eye. 2016;39:38-46

29. Plainis S, Atchison DA, Charman WN. Power profiles of multifocal contact lenses and their interpretation. Optom Vis Sci. 2013;90:1066-1077.

30. Bakaraju RC, Ehrmann K, Ho A, Papas E. Inherent ocular spherical aberration and multifocal contact lens optical performance. Optom Vis Sci. 2010;87:1009-1022.

31. Plainis S, Ntzilepis G, Atchison DA, Charman WN. Through-focus performance with multifocal contact lenses: effect of binocularity, pupil diameter and inherent ocular aberrations. Ophthalmic Physiol Opt. 2013;33:42-50.

32. Guirao A, Williams DR, Cox IG. Effect of rotation and translation on the expected benefit of an ideal method to correct the eye's higher-order aberrations. J Opt Soc Am A. 2001;18:1003-1015.

33. Charman WN, Walsh G. Retinal image quality with different designs of bifocal contact lens. In: Transactions BCLA; 1986.

34. Ehrmann K, Fedtke C, Radic A. Assessment of computer generated vision charts. Cont Lens Anterior Eye. 2009;32:133-140. 
35. Bakaraju RC, Fedtke C, Ehrmann K, Ho A. Comparing the relative peripheral refraction effect of single vision and multifocal contact lenses measured using an autorefractor and an aberrometer: A pilot study. J Optom. 2015;8(3):206-218.

36. Papas EB, Decenzo-Verbeten T, Fonn D, et al; Utility of short-term evaluation of presbyopic contact lens performance. Eye Contact Lens. 2009;35:144-148.
37. Woods J, Woods C, Fonn D. Visual Performance of a Multifocal Contact Lens versus Monovision in Established Presbyopes. Optom Vis Sci. 2015;92:175-182.

\section{Publish your work in this journal}

Clinical Optometry is an international, peer-reviewed, open access journal publishing original research, basic science, clinical and epidemiological studies, reviews and evaluations on clinical optometry. All aspects of patient care are addressed within the journal as well as the practice of optometry including economic and business analyses. Basic and clinical
Dovepress

research papers are published that cover all aspects of optics, refraction and its application to the theory and practice of optometry. The manuscript management system is completely online and includes a very quick and fair peer-review system, which is all easy to use. Visit http://www.dovepress. com/testimonials.php to read real quotes from published authors. 